Review

\title{
The Potential Impact of COVID-19 on Depression and Suicide Risk in Older Adults
}

Stephanie H Gomez ${ }^{1,{ }^{*}{ }^{+}}$, Josephine Ridley ${ }^{1,2,{ }^{+}}$, Silvia C Hernandez ${ }^{1,{ }^{+}}$

1. Department of Psychological Sciences, Case Western Reserve University, Cleveland, OH, USA; EMails: shg64@case.edu; jxr614@case.edu; josephine.ridley@va.gov; scr63@case.edu

2. Veterans Affairs Northeast Ohio Healthcare System, Cleveland, OH, USA.

+ These authors contributed equally to this work.

* Correspondence: Josephine Ridley; E-Mail: josephine.ridley@va.gov; jxr614@case.edu

Academic Editor: Bart Ellenbroek

Special Issue: Depression and Aging

OBM Neurobiology

2021, volume 5 , issue 4

doi:10.21926/obm.neurobiol.2104112
Received: January 19, 2021

Accepted: November 22, 2021

Published: December 24, 2021

\begin{abstract}
COVID-19 infections, hospitalizations, and deaths continue to skyrocket across the world, leaving a trail of enduring psychological turmoil. It wreaks havoc on the physical, emotional, and cognitive health of those directly infected, as well as caretakers, friends, and families. Though the pandemic has at least indirectly impacted most people, older adults appear to be the most vulnerable to COVID-19-related deaths and little is known about the psychological consequences. Unfortunately, many of the precautions taken to minimize infections may have inadvertent negative consequences among older adults. Most recently, researchers have highlighted the need to focus both on preventing the illness and preventing the potential deleterious psychological sequelae of trying to survive a pandemic. In the present paper, past pandemics are examined to hypothesize the likely long-term effects that COVID-19 may have on depression and suicide risk in older adults and to provide guidance on strategies to mitigate the potential negative outcomes.
\end{abstract}

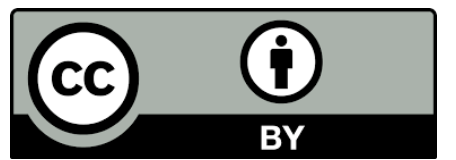

(C) 2021 by the author. This is an open access article distributed under the conditions of the Creative Commons by Attribution License, which permits unrestricted use, distribution, and reproduction in any medium or format, provided the original work is correctly cited. 


\section{Keywords}

COVID-19; pandemic; older adults; depression; suicide

\section{Introduction}

The scientific community has called for an emphasis on researching the current and potential effects of the COVID-19 pandemic on older adults [1], as COVID-19 is disproportionately affecting older people. As of September 2021, 44.4\% of adults aged $60+$ years with a confirmed case of COVID-19 showed symptoms, representing $18.4 \%$ of all confirmed cases with symptoms in the United States [2]. Of those aged 60+ years who were symptomatic and tested positive, $20 \%$ were hospitalized and $8.1 \%$ died as a result of the virus [2]. This is in stark contrast to younger individuals ( $<60$ years), where $3.6 \%$ of cases that were symptomatic and tested positive were hospitalized and $0.3 \%$ died as a result of the virus [2]. To add a greater perspective, it is worth reporting how these numbers compare to the annual flu. In 2016-2017, an estimated $12.5 \%$ of people $65+$ years of age were infected and symptomatic with influenza [3]. However, of the older adults who had symptomatic illnesses, $0.09 \%$ were hospitalized and $0.009 \%$ died as a result [3]. The relative high rate of infection, hospitalization, and death related to COVID-19 paints a grim picture for the mental wellbeing of older adults. The present article will explore past pandemics to hypothesize the likely long-term effects that COVID-19 may have on depression and suicide risk in older adults and provide guidance on strategies to mitigate the potentially deleterious outcomes.

Eight percent of older adults in the general US population experience depression [4], with a 12month prevalence of 5.4\% for Major Depressive Disorder [5]. Moreover, adults aged 65+ years are among the highest at-risk groups for dying by suicide, with rates per 100,000 ranging between 15.19 (65-69 years) and 20.10 (85+ years) [6]. Taken together, males $75+$ years of age are the most at-risk demographic group for suicide, with a rate of 39.9 per 100,000 [7]. Pre-pandemic risk factors for depression and suicide, such as social isolation and loneliness, financial burden, poor physical health, loss of autonomy, alcohol and substance use, and access to lethal means, have intensified during the course of the pandemic [8]. Quarantine, physical distancing, and the minimizing of social and religious gatherings may increase loneliness in older adults [9]. The increased rate of unemployment and general financial instability have likely increased financial anxiety. In the US, firearm purchases increased by $85 \%$ in March 2020 compared to March 2019 [10]. This increase is concerning from a suicide prevention perspective, as nearly half of all suicides in the US in 2018 occurred via firearms [7]. However, help-seeking behaviors (e.g., calls to crisis centers) have also increased [8], which may suggest a certain level of normalization of seeking help for mental health concerns. Moreover, although early data show that depression and suicidal ideation have increased most in younger and not older individuals [11], the long-term consequences of the pandemic among older adults are unknown and need to be well-monitored.

The first aim of the current review is to utilize a historical perspective to shed light on the potential long-term impacts of the COVID-19 pandemic on depression and suicide among older adults. The second aim is to use the historical review to inform current prevention and intervention strategies. 


\section{Impact of Past Pandemics on Depression and Suicide}

Pandemics have an overwhelming impact on the mental health of those affected, both directly and indirectly [12]. While those who contract the disease must deal with the cost of medical care, social isolation, negative stigma, and long-term health consequences, those who do not contract the disease must deal with the anxiety surrounding possible infection and providing care to loved ones who are infected. The mental health of older adults may be uniquely affected by pandemics through magnification of their own distinct risk factors. Looking back on past pandemics can give insight into the long-term mental health consequences of COVID-19 on older adults, specifically in regards to depression and suicide. The mental health effects of past pandemics are reviewed to predict how COVID-19 may impact depression and suicide in older adults.

\subsection{H1N1 Influenza A Virus Outbreak of 1918}

The H1N1 influenza A virus outbreak of 1918, otherwise known as the Spanish flu, was the deadliest pandemic of the $20^{\text {th }}$ century. An estimated 500 million people across the world contracted the disease and over 50 million people died as a result of the disease [13]. Although there is minimal research on the mental health effects of the $1918 \mathrm{flu}$ on the general population, the research that has been conducted points to the pandemic leading to adverse mental health consequences. In an article that discussed various impacts of the 1918 influenza pandemic, Wasserman [14] found a significant association between the mortality rate and the suicide rate in the US during the time of 1918-1920. Specifically, the suicide rate increased during the first phase of the pandemic, when mortality peaked. Wasserman argues that suicide rates tend to decrease during periods of social integration in which there is a unifying of society and an increase in togetherness, as was the case during and after World War I. In contrast, an increase in suicide deaths associated with the 1918 influenza pandemic suggests that there may be unique risk factors attributed to pandemics that negatively affect mental health across society. Pandemics require the implementation of procedures that keep people apart and increase social isolation in order to decrease rates of infection. Many places where people usually obtain social support, such as schools, churches, community-based groups, and large gatherings were generally prohibited. Of note, while Wasserman's research focused on the United States, a recent study did not find a relationship between the 1918 influenza pandemic and suicide rates in Sweden [15]. It is important to acknowledge that data may vary across countries and ongoing assessment of suicide trends is imperative. Nevertheless, pandemics may be particularly detrimental to the mental health of those who are already at increased risk of psychiatric disorders like depression.

Additionally, one study assessed the impact of the 1918 influenza pandemic on asylum hospitalizations in Norway $[16,17]$. They found that the number of patients hospitalized for the first time due to mental illnesses following influenza increased by an annual factor of 7.2 in the six years after the pandemic. The study also reported that those who recovered from the disease reported feelings of depression, sleep disturbances, distraction, dizziness, and coping difficulties. Although the 1918 flu primarily affected younger adults, the mental anguish associated with the deadly pandemic cut across age groups. Ellis and colleagues [18] reference multiple cases of suicide related to the influenza pandemic, emphasizing that the risk should not be solely associated with younger adults. Although there are several limitations to case studies, they can help contextualize factors surrounding the 1918 influenza that may have led someone towards suicidal thoughts and 
behaviors. These findings as a whole point to the severity of the mental health consequences associated with a deadly pandemic, and emphasize the importance of protecting vulnerable populations.

\subsection{Severe Acute Respiratory Syndrome of 2003}

Severe Acute Respiratory Syndrome (SARS-CoV-1 or SARS) is a viral infectious disease that became prominent in 2003 and caused a massive public health crisis across multiple countries [19]. Above the severe physical health effects, SARS is associated with various adverse mental health outcomes. In a sample of 195 SARS survivors, 18\% reported depressive symptoms one month after their hospital discharge, with lack of emotional support being a significant predictor [20]. In regards to more long-term effects, depressive symptoms were moderately to extremely severe in over $40 \%$ of SARS survivors one year after their recovery [21]. Additionally, SARS survivors exhibited elevated stress levels both during the outbreak and one year post outbreak [21]. Furthermore, the negative mental health effects from SARS extend to those who did not contract SARS themselves, but had loved ones who were infected. For example, around $50 \%$ of family members of SARS patients reported psychological problems, including feelings of depression [22]. One study investigated the psychological effects of quarantining among those who had contracted the virus in Canada and found increased rates of depressive and PTSD symptoms [23]. All participants reported a sense of isolation while in quarantine and their psychiatric symptoms increased as duration of quarantine increased. Income was a significant factor as well, with psychiatric symptoms increasing as annual income decreased [23]. These results underscore the importance of considering how various factors can exacerbate the deleterious effects a pandemic has on specific vulnerable populations.

Although many people affected by SARS suffered adverse mental health consequences, it is important to look at the mental health effects specifically in older adults. Most prominently, the older adult suicide rate in Hong Kong showed a significant increase in 2003-2004, which is the same time that SARS emerged as an epidemic in the country $[24,25]$. The older adult suicide rate in Hong Kong was in a downward slope from 1986 to 2002, so the abrupt upward trend is surprising. Furthermore, the number of older adult suicide deaths reached its peak in April 2003, which is when the number of SARS cases reached its peak in the country [25]. Poisson regression models have confirmed that older adult suicides were significantly higher in April 2003 compared to April of other years, and older adults suicide rates were significantly higher in 2003 compared to 2002 [26]. These results are striking, as they point to the possibility of infectious diseases leading to increased suicide rates in older populations.

The mechanisms leading to these worsened mental health outcomes are unknown but can be surmised based on prior research. For example, older adults living in highly infected communities in Hong Kong had significantly lower subjective well-being reports in regards to personal health, personal safety, and future security compared to younger adults [27]. In the same study, the presence of chronic illness and unemployment also had a significant effect on reduced wellbeing, both of which are common in older adults. These findings show that even older adults who do not contract the virus are worse off than younger people who do not contract the virus, particularly in neighborhoods with higher rates of infection. However, an increased sense of communityconnectedness mitigated these effects, pointing to possible targets for intervention. Lee and colleagues [28] found that almost all residents of the Amoy Gardens, the first official site of SARS 
cases, experienced social stigma, including being shunned, insulted, and rejected in various interpersonal domains. This social stigma may be particularly deleterious to the mental health of older adults and supports the idea that increasing community support and decreasing community judgement may prevent depression in older adults infected during a pandemic.

\subsection{H1N1 Pandemic of 2009}

A third pandemic that affected millions across the world was the 2009 H1N1 pandemic, otherwise known as swine flu. There were around 18,500 laboratory-confirmed deaths due to the virus, although the true number of deaths is estimated to be over 200,000 within the first year [29]. There is less research on the mental health consequences of this disease, and the research that has been done finds more associations with anxiety-related symptoms rather than depressive symptoms. In a 2009 telephone survey of 997 UK adults, around one quarter of the sample reported significant anxiety about the swine flu [30]. Additionally, in a survey of over 300 college students during the peak of the swine flu pandemic, anxiety related to swine flu was common [31]. More specifically, health-related anxiety, concerns around contamination, disgust sensitivity, and the tendency to carefully monitor one's internal body sensations were all positively associated with anxiety pertaining to swine flu [31]. Multiple researchers have suggested that this significant anxiety related to swine flu (i.e., "pandemic anxiety") was likely due to its exceptionally wide coverage by news media, politicians, and global health agencies in relation to its anticipated severity [32]. The swine flu did not require a mass quarantine or widespread societal shut down as was present during other pandemics, which may be why depressive symptoms were less prevalent. This information supports the idea that mass quarantining and social isolation may be a significant factor contributing to the increased depressive symptoms seen across populations since the beginning of the COVID19 pandemic. Prior research has shown that quarantining can lead to negative psychological effects such as posttraumatic stress symptoms, confusion, and anger [33]. Given that one quarter of adults 65 and older were considered socially isolated before the COVID-19 pandemic hit [34], this age group is particularly vulnerable to the negative effects of quarantine.

\section{Current Impact of COVID-19 on Depression and Suicide in Older Adults}

Older adults may be at greater risk of suffering from immediate and long-term repercussions as a result of the high rates of losses, loneliness, financial/resource burden, and health complications associated with COVID-19 [35, 36]. A survey completed by 60+ year olds in March 2020 suggested that negative expectations for income decline, the duration of the pandemic, and the long-term impact predicted increased negative affect, with perceived stress as a mediator [36]. A recent study found that COVID-19 was significantly associated with risk of psychiatric illness beyond that which occurs after other acute health events [37]. Depression was the most common mood disorder, associated with a 1.7\% probability of first-time diagnosis for patients with COVID-19 compared to other health events. Older adults with a psychiatric history appear to be at particular risk for contracting COVID-19 compared to younger age groups and to a cohort matched for physical risk factors $(O R=1.25)$, which consequently might increase their vulnerability to developing depression or the worsening of depressive symptoms [37].

With mandates to shelter in place and maintain physical distance, as well as the lower rates of technology and internet access [38] and use [39], older adults have a "double burden of social 
exclusion," meaning exclusion from both physical and digital contact [40]. A study on the impact of social relationships and well-being among older adults re-contacted participants during April/May 2020 to assess the effect of COVID-19 on these variables. Older adults who were sheltering in place experienced more depression (albeit still at a subclinical level) and loneliness after the order was issued compared to baseline, with greater loneliness predicting greater depression [41]. The rate of increase in depression may continue to rise based on the length of the pandemic and the resurgence of physical distance and stay at home orders. Additional consequences include reduced physical activity and limited access to routine health care and/or home care visits [42], which may contribute to the risk of developing or worsening depression and decreased detection by social supports and health professionals.

Findings suggest that, in the context of the pandemic, older adults who are prone to negative thinking patterns, have limited coping abilities, have poor social support, or experience increased loneliness may be particularly vulnerable to developing depression. Ageist beliefs and negative internalized perceptions of aging (i.e., economic burden, dependence) [43] may further intensify negative self-perceptions, depression, hopelessness, and helplessness [44], which may also increase late life suicide risk [45]. However, there is also evidence that older adults are better able to cope with the pandemic compared to younger cohorts [46]. Strengths are associated with resilience, meaning in life, positive life reflection, and generativity (i.e., giving back), which can be used to curb the expected negative consequences related to the pandemic [44,47]. It is also possible that older adults who were not negatively affected by social isolation pre-pandemic do not experience negative psychological effects from quarantining.

Information to date does not indicate an increase in suicide rates due to COVID-19 [48-51], although it is still early to be able to observe such an increase in a relatively rare event [8]. One systematic review found an increased rate of suicidal ideation during the COVID-19 pandemic among the general population, which could potentially lead to increased suicide rates [52]. Other recent systematic reviews have found some support for associations between past epidemics and suicide-related outcomes, though the available research is limited by the quality, quantity, and inconsistency of findings $[53,54]$. Given the lack of research examining the relationship between pandemics and suicide risk, the inconsistent findings of existing research, and the unique factors that may increase suicide risk in older adults, it is important to consider how the COVID-19 pandemic could impact suicide rates in this vulnerable population.

\section{Future Impact of COVID-19 on Depression and Suicide in Older Adults}

It is imperative to focus on how COVID-19 may affect the long-term mental health stability of older adults, as older adults are more likely to be hospitalized for and die by COVID-19. Current observations suggest that COVID-19 infection is significantly associated with the development of depression [37]. To begin, the trajectories of past pandemics and known risk factors support the prediction that COVID-19 could increase rates of depression and suicide in older adults. This is based on evidence that suicide rates increased in the US due to 1918 flu, during which a similar type of mass quarantine was required in order to contain the virus. Additionally, the suicide rate was shown to dramatically increase in Hong Kong, specifically among older adults, during the peak of the SARS pandemic in 2003. Older adults are especially vulnerable during the current COVID-19 pandemic, and therefore the authors believe that older adults will be particularly susceptible to the 
negative psychological effects associated with a pandemic of this kind. They are more likely to have friends and family who are also older and die from COVID-19 and may be less likely to use technology to decrease their social isolation during quarantine. Additionally, it is likely that increased rates of depression and suicide in older adults could persist beyond the duration of the pandemic itself. This is based on the findings of multiple research studies in which the negative psychological effects of the SARS pandemic persisted in survivors one year after their recovery in a significant proportion of the sample. An additional study investigating chronic post-SARS syndrome in 22 individuals indicated the presence of depression, fatigue, and disordered sleep over one year after onset of the illness [55]. Although these symptoms could be a direct result of the virus itself, the investigators acknowledged that the persistence of the symptoms could also be due to the psychological trauma of combating the disease. Finally, older adults who were struggling with mental health problems prior to the COVID-19 pandemic may be at particular risk of developing depressive symptoms or suicidal thoughts as a result of the pandemic. Prior studies found that, during the swine flu pandemic, those who were already struggling with obsessive-compulsive beliefs and health anxiety were more likely to suffer from pandemic-related anxiety [31, 56]. Overall, depression and suicide rates may increase in older adults due to the COVID-19 pandemic. These increases will likely persist beyond the duration of the pandemic itself, and older adults who were already struggling with mental health problems will be particularly vulnerable to these negative effects.

The mechanisms that facilitate these negative mental health consequences can be estimated based on research on past pandemics, recent studies on the psychological effects of COVID-19, and what is already known about risk and protective factors for depression and suicide in older adults. To begin, social isolation will be one of the most significant contributors to the increase in depression in older adults. One study of over 1,000 older adults found that subjective social isolation from family and friends was associated with increased depressive symptoms and psychological distress [57]. Multiple studies have found that suicidal older adults report lower levels of social support and belongingness than non-suicidal older adults [58]. Mass quarantines are usually required during pandemics in order to contain the spread of the disease. Individuals, especially older adults and those with chronic medical issues, are encouraged to stay home during the COVID-19 pandemic due to the risks associated with infection. As a result, many older adults who live alone may go weeks at a time with little to no social interaction, even if they had social support systems in place prior to the pandemic. Older adults may feel a sense of rejection when their younger family members avoid seeing them in person, even though these actions come from a place of love and protection. Additionally, mass quarantines involve the closing of many avenues of social connectedness for older adults, including religious gatherings, community centers, and support groups. Although the pandemic has resulted in many of these social gatherings becoming virtual, many older adults do not have the technological knowledge or comfort to use the required video conferencing platforms [59]. Furthermore, older adults who are living in nursing homes or hospitals are unable to see their family members and friends due to the pandemic restrictions, increasing feelings of isolation and loneliness $[60,61]$. Studies conducted since the beginning of the pandemic indicate that older adults are already reporting increased loneliness that is contributing to feelings of depression [39]. For example, six to ten weeks after the implementation of a visitor ban, older long-term care facility residents showed high levels of depression, loneliness, and an exacerbation 
of mood and behavioral problems [60]. Overall, social isolation and loneliness from the COVID-19 pandemic may lead to increased feelings of depression in older adults in the long term.

The COVID-19 pandemic may also lead to increased suicidal thoughts and behaviors among older adults, which can be understood via the Interpersonal Theory of Suicide. This theory proposes that suicidal desire is created through the simultaneous presence of two key factors: thwarted belongingness and perceived burdensomeness [62]. Thwarted belongingness refers to the state in which an individual's need for social connectedness (i.e., "need to belong") is unmet. Perceived burdensomeness refers to the state in which an individual feels they are a burden on their family members and friends. The Interpersonal Theory of Suicide distinguishes suicidal desire from suicide capability and proposes that both must be present for lethal suicidal behavior to occur, alongside a pervasive sense of hopelessness [62]. Suicide capability may increase from reduced fear of death or increased physical pain tolerance.

In terms of the COVID-19 pandemic, it is very likely that the social isolation and loneliness that older adults experience may increase their thwarted belongingness and therefore increase their risk for suicidal thoughts. A systematic review identified that older adults who engaged in suicidal behaviors reported a sense of losing control, alienation, disconnectedness, and accumulated suffering [63], all of which have been exacerbated by the pandemic. Moreover, the COVID-19 pandemic also has the potential to increase perceived burdensomeness among older adults. Older adults who contract COVID-19 and experience related health problems may feel like an emotional burden on their families, especially if they need to be hospitalized. Additionally, many older adults live with their adult children. These older adults may feel like a financial burden on their families, especially during a time of record high unemployment in the US. Finally, the COVID-19 pandemic has the potential to increase acquired capability for suicide in older adults. Sheffler and colleagues [64] propose that the nonstop media coverage surrounding COVID-19 deaths, as well as the likelihood of personally knowing someone who died from COVID-19, has the capability of desensitizing older adults towards the idea of death and can subsequently reduce their fear of dying. Decreased fear of dying can result in higher capability of suicide and may increase one's risk of acting on suicidal thoughts. Perceived burdensomeness, thwarted belongingness, increased capability for suicide, and the other well-established risk factors for late-life suicide, such as psychiatric disorders, hopelessness, and physical illness, should alert health providers and community members to increase screening and monitoring of suicidal thoughts and behaviors in older adults.

In sum, COVID-19 has the potential to increase depression and suicide in older adults in the long term (see Table 1). Mass quarantining has already increased social isolation and feelings of loneliness among older adults, and this may turn into feelings of depression as the pandemic persists. Additionally, the COVID-19 pandemic has the potential to increase thwarted belongingness, perceived burdensomeness, and acquired capability for suicide amongst older adults. Based on the Interpersonal Theory of Suicide, these factors could lead to increased suicide rates among older adults if we do not take preventative action as soon as possible. Fortunately, if communities take precautionary steps now, we may be able to promote the psychological resilience older adults need to avoid these fatal outcomes. 
Table 1 Summary of COVID-19 pandemic factors, mediating variables, and expected negative outcomes for older adults.

\begin{tabular}{lll}
\hline COVID-19 Pandemic Factors & Mediating Variables & Outcomes \\
\hline $\begin{array}{l}\text { Higher rates of infection, } \\
\text { hospitalization, and death }\end{array}$ & Quarantining & Higher rate of grief \\
Higher rate of loss & Limitations on social gatherings & $\begin{array}{l}\text { Increased isolation and } \\
\text { loneliness }\end{array}$ \\
& $\begin{array}{l}\text { Lower rates of technology access } \\
\text { and use for socialization }\end{array}$ & Increased stress \\
& Increased medical bills & Increased financial burden \\
& & \\
&
\end{tabular}

\section{Past Interventions}

Prior pandemics have resulted in an increase in suicide attempts, depression and anxiety, as well as a myriad of other physical and mental health problems [65]. However, empirical studies examining methods for reducing these negative mental health effects are sparse. The approaches documented in the few existing studies can be described as public health oriented, with a focus on mental health providers and public policies, all with the intent of mitigating the psychological damage of the pandemics. Most studies focused on the general population, healthcare workers, or specific vulnerable populations, but very few focused on the older population. Two existing systematic reviews provided comprehensive overviews of psychological interventions in response to mental health problems following pandemics or epidemics.

One systematic review utilized PubMed, Web of Science, Embase, PsychINFO, and the WHO Global Research Database on COVID-19 to search for articles focused on psychological interventions used during infectious disease outbreaks [66]. The authors identified 32 studies that examined psychological interventions for mental health problems related to epidemics or pandemics. Specifically, 23 articles focused on COVID-19, seven on Ebola, one on Middle East respiratory syndrome (MERS), and one on SARS, with interventions categorized into mental healthcare systems, psychosocial interventions, psychiatric hospital responses, and digital mental healthcare. However, only one study they reviewed addressed older adults [67]. The authors concluded that local and national-based strategies, including the provision of evidence-based interventions (e.g., cognitivebehavioral therapy and psychological first aid), and community and culturally adapted interventions, resulted in positive outcomes when applied to communities affected by SARS, MERS, Ebola, and 
COVID-19 [66]. They found the use of telehealth services central to successfully responding to mental health needs following outbreaks.

Brooks and colleagues [33] conducted a rapid review of the literature on ways to mitigate the psychological consequences of quarantining during infectious disease outbreaks. The authors found evidence of multiple strategies to lessen the negative effects of quarantine. First, based on the research linking length of quarantine with greater psychological distress, they recommended restricting the length of the quarantine [68]. Second, research suggested that providing as much information as possible to the public and avoiding sensationalism by the media could be helpful, as well as providing access to basic necessities for activities of daily living [69]. Third, one study found that providing technology in the form of mobile phones, charging tools, and adequate Wi-Fi networks reduced feelings of panic, isolation, and stress by improving connectedness and relieving boredom [70]. Furthermore, there is evidence for the utility of support groups designed specifically for individuals required to quarantine at home [71]. The results showed an improvement in feeling connected to others having the same experiences, and feeling validated, empowered, and supported. The authors' final recommendation was based on several studies that showed appealing to individuals' altruism was effective in maintaining wellbeing and reducing the stress of quarantine $[72,73]$. These studies demonstrated that the genuinely expressed appreciation from the authorities for their quarantine compliance, as well as an emphasis on the results of their efforts to keep vulnerable populations safe, resulted in improved cooperation and reduced negative mental health outcomes.

\section{Current Mitigating Strategies}

Multiple factors have been identified that may contribute to increased anxiety, depression, and potential suicide in the older population due to the COVID-19 pandemic. Protective factors such as resilience, meaning in life, positive life reflection, and generativity have also been identified [47]. A call for "urgent action" to mitigate the predicted negative psychological consequences of forced social isolation among older adults was made by multiple researchers [74-76]. Holmes and colleagues [74] quickly gathered an interdisciplinary group of 24 individuals to make recommendations on how to mitigate the effects of COVID-19 on the psychological health of vulnerable groups. They identified the need for population-specific interventions, policy and public health strategies, increased funding, and evidence-based interventions that include technology and non-technology based approaches.

In an editorial focused on reducing the psychological impact of COVID-19, Moutier [8] categorized what she termed "threats to suicide risk" into eight groups, along with recommended strategies for mitigating each risk factor. The first category consisted of mental illness and suggested the use of healthcare systems, individual clinicians, and government support of the Zero Suicide framework, as the most likely solutions. The other seven categories of threats to suicide risk included: 1) isolation/loneliness/bereavement; 2) suicidal crisis; 3) alcohol consumption; 4) exposure to increased domestic violence; 5) financial strain; 6) exposure to irresponsible media reporting; and 7) access to lethal means. To mitigate these risks, she recommended increasing access to mental health services and training providers to use Counseling on Access to Lethal Means (CALM) and other evidence-based interventions. Additional recommendations included improving 
public messaging, public policies, crisis hotlines, organizational commitment and support, and interpersonal engagement.

Reger, Stanley, and Joiner [35] identified similar general population suicide risk factors related to COVID-19 and multiple public health interventions to address those risks. First, they recommended distinguishing between physical distancing and social distancing. Rather than using the terms "social distancing," messaging should maintain the focus on keeping a physical distance while staying connected. The use of telehealth for the provision of psychotherapy and other interventions is strongly recommended to help reduce suicide risk and related factors. They noted that internet and computer access must be addressed to make this an effective solution; and this may be especially true for rural areas. An increase in screening and monitoring for crises would be needed for individuals whose access to traditional mental healthcare has been limited. Furthermore, they recommended increasing the use of "distance-based suicide prevention" methods such as caring contact letters and telephone-based outreach. Finally, they address media reporting of suicide events. Specifically, media is directed to follow the reporting guidelines recommended by suicide prevention agencies (e.g., American Association of Suicidology guidelines) and include the national suicide prevention crisis line and/or local crisis resources.

\section{Recommendations}

By far, research has indicated that one of the strongest risk factors for depression and suicide amongst older adults is social isolation and its consequent loneliness [45, 76]. The stress that arises from isolation and loneliness has also been linked to health risks [77]. Social distancing, while mandatory and lifesaving, has the paradoxical effect of increasing physical isolation and feelings of loneliness, which increases the potential for thwarted belongingness and perceived burdensomeness [62]. Fortunately, decades of research have helped to identify protective factors and effective suicide prevention strategies. Targeting the specific aspects of the pandemic that increase suicide and depression risk among the older population may be the best approach.

\subsection{Technology-Based Interventions}

Individual and group video chats with friends and family, as well as religious services offered via video live streaming, can increase social connectedness among older adults. Additionally, the use and dissemination of virtual peer support and social groups aimed at fostering social connection among older adults may be beneficial. For example, Covia.org offers free phone or video-based weekly groups aimed at connecting older adults with similar interests, as well as participating in a range of classes. Similar services could be developed for older caregivers and family members who are caring for another individual infected with COVID-19.

However, the heavy reliance on technology may present as a barrier to many older adults who are not comfortable using technology. Most of the research looking at the use of telehealth among older adults has shown positive outcomes with some challenges. A major problem is access to the internet and the bandwidth capable of allowing the use of platforms such as Zoom, Webex, or Microsoft Teams. Another challenge is access to technological tools such as smartphones, tablets, or laptops. These materials can be cost prohibitive and difficult to operate for many seniors. Thus, barriers to the use of technology to overcome social isolation include lack of resources and skills in using technology. 
Potential solutions may come as public health approaches, such as offering incentives to major corporations to provide free or low-cost mobile devices to older adults. Internet and cell phone companies could also be incentivized to supply internet services with sufficient bandwidth to support video connections for older adults. Campaigns to educate older individuals in the use of technology have also shown promise [8]. Furthermore, agencies can provide socially distanced contact with seniors to provide training in the use of smartphones and laptops. Volunteer organizations can offer assistance in training and delivering devices to those in quarantine. This also provides a much needed opportunity for interaction and moments of connectedness that may reduce the negative impact of social isolation.

\subsection{Education and Information Strategies}

Overall, the tone across public messaging should be one of cohesion and community togetherness in the face of adversity, as an increased sense of community has been observed to mitigate negative mental health effects. Public Service Announcements focused on educating the public about the benefits of connecting via video technologies and providing basic instructions may be useful. An additional benefit of education-based initiatives is the possibility of increasing selfefficacy and technological literacy in older populations. In general, public messaging should focus on empowering older adults by reinforcing their ability to learn and benefit from technology. Learning new skills and feeling empowered to adapt to changes can be a powerful protective factor against the development or reinforcement of negative age-related perceptions or a blunted selfesteem. Testimonials by older adults could also be used to increase public messaging effectiveness. However, many older adults are already adept at using technology, and public service announcements should not always assume that older age is synonymous to technological illiteracy.

Increasing public messaging and advertisement campaigns to specifically target older adults can help promote help seeking behaviors for their mental health needs and provide general support. Given the high rate of deaths related to COVID-19 among older adults, public messaging should also focus on psychoeducation about the bereavement and healing process. Support lines should be included with all messaging to facilitate connection with resources.

Finally, provider education may help to mitigate the pandemic-related increased risk of depression and suicide among older adults. Providing direct training to mental health and nonmental health providers on suicide risk in older adults may result in better patient engagement, positive beliefs about aging, and overall improvements in provider ability to assess and manage geriatric suicide risk [45]. Arming providers with the necessary skills and knowledge to work with the older population will decrease the potential negative impact of myths about aging that may interfere with the provision of adequate care for this population.

\subsection{Evidence-Based Interventions and Other Strategies}

In addition to the approaches mentioned above, there are multiple evidence-based practices that can be implemented to mitigate depression and suicide risk in older adults during pandemics. For example, Caring Contacts (CC) is an empirically-supported intervention for reducing suicide risk [78] that has been effectively implemented in many countries with the use of volunteers and nonmental health agencies. Adopt-a-grandparent programs by neighboring schools have shown promise for reducing the loneliness and disconnectedness of nursing home residents, while 
improving their meaning in life and sense of purpose. For those already struggling with suicidal thoughts, safety plans can be developed with trained individuals to keep older adults safe. Multiple safety planning applications can be accessed on mobile devices that can connect to identified support contacts and crisis phone numbers. Crisis support telephone numbers, online chat websites, and crisis text lines, can be mailed to each household in the form of refrigerator magnets or other easily seen materials. Finally, pharmacological interventions, especially antidepressants, have been shown to be effective in reducing depression and suicidal thoughts and behavior in geriatric populations [78].

Statistically, older adults are more likely to know someone their age who contracted COVID-19 and suffered severe consequences or died as a result. Efforts should also focus on processing grief and loss [79]. Support groups for coping with COVID-19-related loss may be one example of creating space for connection and validation of difficult experiences, as well as mitigating risks for depression and suicide risk. Healthcare providers can learn about the grieving process, offer empathetic listening to their patients, and promote healthy adaptations to loss [79].

As the pandemic progresses and vaccinations become publicly distributed, older adults must remain at the center of policy, healthcare, and local-level agendas. Ongoing work will need to take place throughout the course of the pandemic, as well as after it has subsided, with aims to foster connectedness, hope, and meaning in life among the most vulnerable.

\section{Conclusions}

By reviewing the existing literature on historical pandemics, the authors identified possible longterm mental health outcomes and interventions aimed at older adults living through the COVID-19 pandemic. Evidence from the SARS pandemic suggests the likelihood of increased stress, depression, and loneliness both during and beyond the pandemic crisis. Depressive symptoms were associated with lack of emotional support, quarantine, and financial insecurity. Though less evidence is available to assess the effect of pandemics on late life suicide, there is suggestive evidence for increased death rates concurrent to the SARS and 1918 Influenza outbreaks. Some evidence suggests that the absence of quarantine may have contributed to a lower prevalence rate of depression and suicide during the swine flu compared to SARS. There is some data that suggests similar mental health outcomes among older adults associated with COVID-19, such as increased depression, stress, and loneliness. The relatively brief time frame to examine a rare event, such as suicide, means that suicide risk should be closely monitored over time.

The encouraging news is that we can use historical and current data to intervene and curb the potential increases in clinical depression and suicide deaths among older adults. Past successful interventions included increased screening of mental health issues, stronger referral systems through community organizations, psychoeducation on the illness, problem-solving therapy, support lines and groups, creative use of technology to foster connectedness, and reframing the need to quarantine as a necessary means to help others stay safe. Similar interventions can be used to combat the long-term effects of COVID-19 on older adult depression and suicide risk. A concerted effort across disciplines, as well as federal, state, and local agencies, is imperative to combat the detrimental long-term effects of the COVID-19 global pandemic among older adults at risk for isolation, loneliness, depression, and suicide. 


\section{Acknowledgments}

We thank VA Northeast Ohio Healthcare System and Case Western Reserve University for their support.

\section{Author Contributions}

$\mathrm{JR}, \mathrm{SH}, \mathrm{SG}$ each contributed to the conception, literature review, drafting, and revising of the article equally. We approve of the version of the manuscript to be published.

\section{Competing Interests}

The authors have declared that no competing interests exist.

\section{References}

1. Vahia IV, Blazer DG, Smith GS, Karp JF, Steffens DC, Forester BP, et al. COVID-19, mental health and aging: A need for new knowledge to bridge science and service. Am J Geriatr Psychiatry. 2020; 28: 695-697.

2. Centers for Disease Control and Prevention. COVID-19 case surveillance public use data [Internet]. Atlanta: Centers for Disease Control and Prevention; 2021. Available from: https://data.cdc.gov/Case-Surveillance/COVID-19-Case-Surveillance-Public-Use-Data/vbimakqf/data.

3. Centers for Disease Control and Prevention. Burden estimates for the 2016-2017 influenza season [Internet]. Atlanta: Centers for Disease Control and Prevention; 2019. Available from: https://www.cdc.gov/flu/about/burden/2016-2017.html.

4. Brody DJ, Pratt L, Hughes J. Prevalence of depression among adults aged 20 and over: United States, 2013-2016. NCHS. 2018; 303: 1-8.

5. Hasin DS, Sarvet AL, Meyers JL, Saha TD, Ruan WJ, Stohl M, et al. Epidemiology of adult DSM-5 major depressive disorder and its specifiers in the United States. JAMA Psychiatry. 2018; 75: 336-346.

6. Centers for Disease Control and Prevention. Web-based injury statistics query and reporting system (WISQARS) [Internet]. Atlanta: National Center for Injury Prevention and Control; 2020. Available from: https://www.cdc.gov/injury/wisqars/index.html.

7. National Institute of Mental Health. Suicide [Internet]. Bethesda: National Institute of Mental Health; 2021. Available from: https://www.nimh.nih.gov/health/statistics/suicide.shtml.

8. Moutier C. Suicide prevention in the COVID-19 era: Transforming threat into opportunity. JAMA Psychiatry. 2021; 78: 433-438.

9. Berg-Weger M, Morley JE. Loneliness and social isolation in older adults during the COVID-19 pandemic: Implications for gerontological social work. J Nutr Health Aging. 2020; 24: 456-458.

10. Small Arms Analytics and Forecasting. US firearms sales: March 2020 unit sales show anticipated covid-19-related boom [Internet]. Greenville: Small Arms Analytics and Forecasting; 2020. Available from: http://smallarmsanalytics.com/v1/pr/2020-04-01.pdf.

11. Czeisler MÉ, Lane RI, Petrosky E, Wiley JF, Christensen A, Njai R, et al. Mental health, substance use, and suicidal ideation during the COVID-19 pandemic-United States, June 24-30, 2020. MMWR. 2020; 69: 1049-1057. 
12. Madhav N, Oppenheim B, Gallivan M, Mulembakani P, Rubin E, Wolfe N. Pandemics: Risks, impacts, and mitigation. In: Disease control priorities: Improving health and reducing poverty. 3th ed. Washington, DC: The World Bank; 2017.

13. Nickol ME, Kindrachuk J. A year of terror and a century of reflection: Perspectives on the great influenza pandemic of 1918-1919. BMC Infect Dis. 2019; 19: 1-10.

14. Wasserman IM. The impact of epidemic, war, prohibition and media on suicide: United States, 1910-1920. Suicide Life Threat Behav. 1992; 22: 240-254.

15. Rück C, Mataix-Cols D, Malki K, Adler M, Flygare O, Runeson B, et al. Will the COVID-19 pandemic lead to a tsunami of suicides? A swedish nationwide analysis of historical and 2020 data. MedRxiv. 2020. doi: 10.1101/2020.12.10.20244699.

16. Eghigian $G$. The spanish flu pandemic and mental health: A historical perspective. Psychiatr Times. 2020; 37: 26.

17. Mamelund SE. Effects of the Spanish influenza pandemic of 1918-19 on later life mortality of Norwegian cohorts born about 1900. Oslo: University of Oslo; 2003.

18. Ellis R, Wynter R, Light R. Body and mind: Are we adequately prepared for the toll this pandemic will take on mental health? Hist Today. 2020; 70: 90-93.

19. Syed Q, Sopwith W, Regan M, Bellis MA. Behind the mask. Journey through an epidemic: Some observations of contrasting public health responses to SARS. J Epidemiol Community Health. 2003; 57: 855-866.

20. Wu KK, Chan SK, Ma TM. Posttraumatic stress, anxiety, and depression in survivors of severe acute respiratory syndrome (SARS). J Traum Stress. 2005; 18: 39-42.

21. Lee AM, Wong JG, McAlonan GM, Cheung V, Cheung C, Sham PC, et al. Stress and psychological distress among SARS survivors 1 year after the outbreak. Can J Psychiatry. 2007; 52: 233-240.

22. Tsang H, Scudds RJ, Chan E. Psychosocial impact of SARS. Emerg Infect Dis. 2004; 10: 1326-1328.

23. Hawryluck L, Gold WL, Robinson S, Pogorski S, Galea S, Styra R. SARS control and psychological effects of quarantine, Toronto, Canada. Emerging Infect Dis. 2004; 10: 1206-1212.

24. Chan SM, Chiu FK, Lam CW, Leung PY, Conwell Y. Elderly suicide and the 2003 SARS epidemic in Hong Kong. Int J Geriatr Psychiatry. 2006; 21: 113-118.

25. Chan SS, Lam LC, Chiu HF. The emergence of the novel H1N1 virus: Implications for global mental health. Int Psychogeriatr. 2009; 21: 987-989.

26. Cheung YT, Chau PH, Yip PS. A revisit on older adults suicides and Severe Acute Respiratory Syndrome (SARS) epidemic in Hong Kong. Int J Geriatr Psychiatry. 2008; 23: 1231-1238.

27. Lau AL, Chi I, Cummins RA, Lee TM, Chou KL, Chung LW. The SARS (Severe Acute Respiratory Syndrome) pandemic in Hong Kong: Effects on the subjective wellbeing of elderly and younger people. Aging Ment Health. 2008; 12: 746-760.

28. Lee S, Chan LY, Chau AM, Kwok KP, Kleinman A. The experience of SARS-related stigma at Amoy Gardens. Soc Sci Med. 2005; 61: 2038-2046.

29. Dawood FS, luliano AD, Reed C, Meltzer MI, Shay DK, Cheng PY, et al. Estimated global mortality associated with the first 12 months of 2009 pandemic influenza a H1N1 virus circulation: A modelling study. Lancet Infect Dis. 2012; 12: 687-695.

30. Rubin G, Amlôt R, Page L, Wessely S. Public perceptions, anxiety, and behaviour change in relation to the swine flu outbreak: Cross sectional telephone survey. BMJ. 2009; 339: b2651.

31. Wheaton MG, Abramowitz JS, Berman NC, Fabricant LE, Olatunji BO. Psychological predictors of anxiety in response to the H1N1 (swine flu) pandemic. Cognit Ther Res. 2012; 36: 210-218. 
32. Everts J. Announcing swine flu and the Interpretation of pandemic anxiety. Antipode. 2013; 45: 809-825.

33. Brooks SK, Webster RK, Smith LE, Woodland L, Wessely S, Greenberg N, et al. The psychological impact of quarantine and how to reduce it: Rapid review of the evidence. Lancet. 2020; 395: 912-920.

34. National Academies of Sciences, Engineering and Medicine, Division of Behavioral and Social Sciences and Education, Health and Medicine Division, Board on Health Sciences Policy, Board on Behavioral, et al. Social isolation and loneliness in older adults: Opportunities for the health care system. Washington, DC: National Academies Press; 2020.

35. Reger MA, Stanley IH, Joiner TE. Suicide mortality and coronavirus disease 2019-a perfect storm? JAMA Psychiatry. 2020; 77: 1093-1094.

36. Whitehead BR. COVID-19 as a stressor: Pandemic expectations, perceived stress and negative affect in older adults. J Gerontol B Psychol Sci Soc Sci. 2021; 76: e59-e64.

37. Taquet M, Luciano S, Geddes JR, Harrison PJ. Bidirectional associations between COVID-19 and psychiatric disorder: Retrospective cohort studies of 62,354 COVID-19 cases in the USA. Lancet Psychiatry. 2021; 8: 130-140.

38. Hunsaker A, Hargittai E. A review of Internet use among older adults. New Media Soc. 2018; 20: 3937-3954.

39. Vogels EA. From virtual parties to ordering food, how Americans are using the internet during COVID-19 [Internet]. Washington: Pew Research Center; 2020. Available from: https://www.pewresearch.org/fact-tank/2020/04/30/from-virtual-parties-to-ordering-foodhow-americans-are-using-the-internet-during-covid-19/.

40. Seifert A, Cotten SR, Xie B. A double burden of exclusion? Digital and social exclusion of older adults in times of COVID-19. J Gerontol B Psychol Sci Soc Sci. 2021; 76: e99-e103.

41. Krendl AC, Perry BL. The impact of sheltering in place during the COVID-19 pandemic on older adults' social and mental well-being. J Gerontol B Psychol Sci Soc Sci. 2021; 76: e53-e58.

42. Schrack JA, Wanigatunga AA, Juraschek SP. After the COVID-19 pandemic: The next wave of health challenges for older adults. J Gerontol A Biol Sci Med Sci. 2020;75: e121-e122.

43. Barrett AE, Michael C, Padavic I. Calculated ageism: Generational sacrifice as a response to the COVID-19 pandemic. J Gerontol B Psychol Sci Soc Sci. 2021; 76: e201-e205.

44. Flett GL, Heisel MJ. Aging and feeling valued versus expendable during the COVID-19 pandemic and beyond: A review and commentary of why mattering is fundamental to the health and wellbeing of older adults. Int J Ment Health Addict. 2020. doi: 10.1007/s11469-020-00339-4.

45. Wand AP, Zhong BL, Chiu HF, Draper B, Leo DD. COVID-19: The implications for suicide in older adults. Int Psychogeriatr. 2020; 32: 1225-3120.

46. Carney AK, Graf AS, Hudson G, Wilson E. Age moderates perceived COVID-19 disruption on wellbeing. Gerontologist. 2021; 61: 30-35.

47. Lind M, Bluck S, McAdams DP. More vulnerable? The life story approach highlights older people's potential for strength during the pandemic. J Gerontol B Psychol Sci Soc Sci. 2021; 76: e45-e48.

48. John A, Eyles E, Webb RT, Okolie C, Schmidt L, Arensman E, et al. The impact of the COVID-19 pandemic on self-harm and suicidal behaviour: Update of living systematic review. F1000Res. 2020; 9: 1097. 
49. John A, Pirkis J, Gunnell D, Appleby L, Morrissey J. Trends in suicide during the COVID-19 pandemic. BMJ. 2020; 371: m4352.

50. Pirkis J, John A, Shin S, DelPozo-Banos M, Arya V, Analuisa-Aguilar P, et al. Suicide trends in the early months of the COVID-19 pandemic: An interrupted time-series analysis of preliminary data from 21 countries. Lancet Psychiatry. 2021; 8: 579-588.

51. Knipe D, John A, Padmanathan P, Eyles E, Dekel D, Higgins JP, et al. Suicide and self-harm in lowand middle- income countries during the COVID-19 pandemic: A systematic review. MedRxiv. 2021. doi: 10.1101/2021.09.03.21263083.

52. Farooq S, Tunmore J, Ali W, Ayub M. Suicide, self-harm and suicidal ideation during COVID-19: A systematic review. Psychiatry Res. 2021; 306: 114228.

53. Rogers JP, Chesney E, Oliver D, Begum N, Saini A, Wang S, et al. Suicide, self-harm and thoughts of suicide or self-harm in infectious disease epidemics: A systematic review and meta-analysis. Epidemiol Psychiatr Sci. 2021; 30: e32.

54. Zortea TC, Brenna CT, Joyce M, McClelland H, Tippett M, Tran MM, et al. The impact of infectious disease-related public health emergencies on suicide, suicidal behavior, and suicidal thoughts. Crisis. 2020; 42. doi: 10.1027/0227-5910/a000753.

55. Moldofsky $\mathrm{H}$, Patcai J. Chronic widespread musculoskeletal pain, fatigue, depression and disordered sleep in chronic post-SARS syndrome; a case-controlled study. BMC Neurol. 2011; 11: 1-7.

56. Brand J, McKay D, Wheaton MG, Abramowitz JS. The relationship between obsessive compulsive beliefs and symptoms, anxiety and disgust sensitivity, and swine flu fears. J Obsessive Compuls Relat Disord. 2013; 2: 200-206.

57. Taylor HO, Taylor RJ, Nguyen AW, Chatters L. Social isolation, depression, and psychological distress among older adults. J Aging Health. 2018; 30: 229-246.

58. Conejero I, Olié E, Courtet $P$, Calati R. Suicide in older adults: Current perspectives. Clin Interv Aging. 2018; 13: 691-699.

59. Smith A. Older adults and technology use [Internet]. Washington: Pew Research Center; 2014. Available from: https://www.pewresearch.org/internet/2014/04/03/older-adults-andtechnology-use/.

60. Simard J, Volicer L. Loneliness and isolation in long-term care and the COVID-19 pandemic. J Am Med Dir Assoc. 2020; 21: 966-967.

61. Trabucchi M, Leo DD. Nursing homes or besieged castles: COVID-19 in northern Italy. Lancet Psychiatry. 2020; 7: 387-388.

62. Orden KA, Witte TK, Cukrowicz KC, Braithwaite S, Selby EA, Joiner TE. The interpersonal theory of suicide. Psychol Rev. 2010; 117: 575-600.

63. Wand AP, Peisah C, Draper B, Brodaty H. Understanding self-harm in older people: A systematic review of qualitative studies. Aging Ment Health. 2018; 22: 289-298.

64. Sheffler JL, Joiner TE, Sachs-Ericsson NJ. The interpersonal and psychological impacts of COVID19 on risk for late-life suicide. Gerontologist. 2021; 61: 23-29.

65. Yip PS, Cheung YT, Chau PH, Law YW. The impact of epidemic outbreak: The case of Severe Acute Respiratory Syndrome (SARS) and suicide among older adults in Hong Kong. Crisis. 2010; 31: 86-92.

66. Yue JL, Yan W, Sun YK, Yuan K, Su SZ, Han Y, et al. Mental health services for infectious disease outbreaks including COVID-19: A rapid systematic review. Psychol Med. 2020; 50: 2498-2513. 
67. Wang $\mathrm{H}, \mathrm{Li}$ T, Gauthier $\mathrm{S}, \mathrm{Yu}$ E, Tang $\mathrm{Y}$, Barbarino $\mathrm{P}$, et al. Coronavirus epidemic and geriatric mental healthcare in China: How a coordinated response by professional organizations helped older adults during an unprecedented crisis. Int Psychogeriatr. 2020; 32: 1117-1120.

68. Rona RJ, Fear NT, Hull L, Greenberg N, Earnshaw M, Hotopf M, et al. Mental health consequences of overstretch in the UK armed forces: First phase of a cohort study. BMJ. 2007; 335: 603.

69. Rubin GJ, Harper S, Williams PD, Öström S, Bredbere S, Amlôt R, et al. How to support staff deploying on overseas humanitarian work: $A$ qualitative analysis of responder views about the 2014/15 West African Ebola outbreak. Eur J Psychotraumatol. 2016; 7: 30933.

70. Manuell ME, Cukor J. Mother nature versus human nature: Public compliance with evacuation and quarantine. Disasters. 2011; 35: 417-442.

71. Pan PJ, Chang SH, Yu YY. A support group for home-quarantined college students exposed to SARS: Learning from practice. J Spec Group Work. 2005; 30: 363-374.

72. Wu P, Fang Y, Guan Z, Fan B, Kong J, Yao Z, et al. The psychological impact of the SARS epidemic on hospital employees in China: Exposure, risk perception and altruistic acceptance of risk. Can J Psychiatry. 2009; 54: 302-311.

73. Armitage R, Nellums LB. COVID-19 and the consequences of isolating the elderly. Lancet Public Health. 2020; 5: e256.

74. Holmes EA, O'Connor RC, Perry VH, Tracey I, Wessely S, Arseneault L, et al. Multidisciplinary research priorities for the COVID-19 pandemic: A call for action for mental health science. Lancet Psychiatry. 2020; 7: 547-560.

75. Niederkrotenthaler T, Gunnell D, Arensman E, Pirkis J, Appleby L, Hawton K, et al. Suicide research, prevention, and COVID-19: Towards a global response and the establishment of an international research collaboration. Crisis. 2020; 41: 321-330.

76. Gerst-Emerson K, Jayawardhana J. Loneliness as a public health issue: The impact of loneliness on health care utilization among older adults. Am J Public Health. 2015; 105: 1013-1019.

77. Luxton DD, June JD, Comtois KA. Can postdischarge follow-up contacts prevent suicide and suicidal behavior? A review of the evidence. Crisis. 2013; 34: 32-41.

78. Wasserman D, losue M, Wuestefeld A, Carli V. Adaptation of evidence-based suicide prevention strategies during and after the COVID-19 pandemic. World Psychiatry. 2020; 19: 294-306.

79. Goveas JS, Shear MK. Grief and the COVID-19 pandemic in older adults. Am J Geriatr Psychiatry. 2020; 28: 1119-1125.

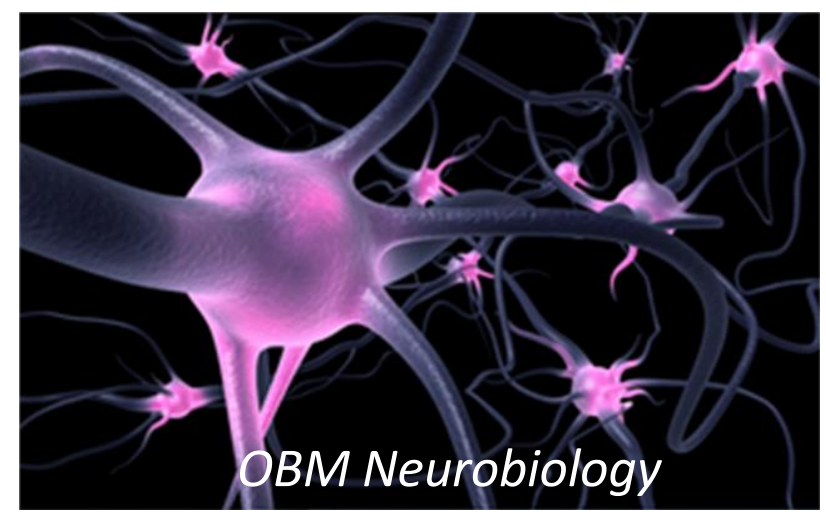

Enjoy OBM Neurobiology by:

1. Submitting a manuscript

2. Joining volunteer reviewer bank

3. Joining Editorial Board

4. Guest editing a special issue

For more details, please visit: http://www.lidsen.com/journals/neurobiology 